\title{
A Generalisation of the Nonlinear Small-Gain Theorem for Systems with Abstract Initial Conditions
}

\author{
Jing Liu and Mark French
}

\begin{abstract}
We consider the development of a general nonlinear small-gain theorem for systems with abstract initial conditions. Systems are defined in a set theoretic manner from input-output pairs on a doubly infinite time axis, and a general construction of the initial conditions (i.e. a state at time zero) is given in terms of an equivalence class of trajectories on the negative time axis. By using this formulation, an ISStype nonlinear small-gain theorem is established with complete disconnection between the stability property and the existence, uniqueness properties. We provide an illustrative example.
\end{abstract}

\section{INTRODUCTION}

The use of the small-gain theorem in control theory dates back to the 1960s by [23] and [16]. The original version of the small-gain theorem involves systems with finite linear gains from input to output with or without a bias term (see e.g., [3]). Extensions of the small-gain theorem to nonlinear gains have been studied by many researchers. The work on the small-gain theory involving nonlinear gains began with [6], [13], where the monotone gain was proposed for a nonlinear generalisation of the classical small-gain theorem. In [9], the authors developed a nonlinear ISS-type small-gain theorem in the sense of [18] for interconnection of nonlinear systems in state space representations, which led an extensive follow-up literature (e.g., [1], [7], [8]). Several interesting extensions of the small-gain theorem were also obtained for systems with special structures such as Volterra systems [24], general networks [2], large-scale complex systems [10], stochastic systems [12], hybrid systems [11], [14], etc.

Note that the classical small-gain theorem obtained in the input-output framework has the benefit that the stability property is completely disconnected from the existence, uniqueness properties, etc (see e.g., [3]). Most of the results of the ISS-type nonlinear small-gain theorem were obtained for nonlinear state space models, and a priori requirements of existence and uniqueness properties of systems are imposed (e.g., requiring smoothness or Lipschitz continuity of dynamical functions), and extra "observability" conditions are imposed to guarantee that the state trajectories are bounded when the input and output are bounded.

One major contribution of this article is that we provide a uniform framework to study input-output theory incorporating abstract initial conditions. Both systems and the corresponding initial conditions are defined from a set theoretic manner. No special structures such as state space model, Volterra series representation are required. Another

J. Liu is with the School of Electronics and Computer Science, University of Southampton, SO17 1BJ, UK, jl4g10 decs.soton.ac.uk

M. French is with the School of Electronics and Computer Science, University of Southampton, SO17 1BJ, UK, mcf@ecs. soton. ac.uk contribution of this article is that we present a nonlinear ISStype small-gain theorem without the extra "observability" conditions and with complete disconnection between the stability property and the existence, uniqueness properties. The main idea of the proof is motivated by [9]. On one hand this can be reviewed as a generalisation of the inputoutput framework to incorporate initial conditions, and on the other hand a generalisation of the ISS/IOS framework to incorporate more general system classes.

The paper is organised as follows. In $\S I I$, we introduce definitions of systems and initial conditions, which involve only input-output structures. In $\S$ III, we present our main result in this paper and the corresponding proof, and give an illustrative example. We draw a conclusion in $\S \mathrm{IV}$.

\section{SYSTEMS AND INITIAL CONDITIONS}

Let $\mathcal{S}$ denote the set of all locally integrable maps $\mathbb{R} \rightarrow \mathcal{X}$ where $\mathcal{X}$ is a nonempty set. For any interval $J$, we regard $\mathcal{S}_{J}$ as a subspace of $\mathcal{S}$ by identifying $\mathcal{S}_{J}$ with the set of maps in $\mathcal{S}$ which vanish outside of $J$. We define a truncation operator $T_{J}: \mathcal{S} \rightarrow \mathcal{S}$ and a restriction operator $R_{J}: \mathcal{S}_{I} \rightarrow \mathcal{S}_{J}$ with $J \subset I$ as follows:

$$
\begin{aligned}
& T_{J}: \mathcal{S} \rightarrow \mathcal{S}, v \mapsto T_{J} v \triangleq\left(t \mapsto\left\{\begin{array}{cc}
v(t), & t \in J \\
0, & \text { otherwise }
\end{array}\right)\right. \\
& R_{J}: \mathcal{S}_{I} \rightarrow \mathcal{S}_{J}, \quad v \mapsto R_{J} v \triangleq(t \mapsto v(t), \quad t \in J) .
\end{aligned}
$$

We let $R_{+} \triangleq R_{[0, \infty)}$ and $R_{-} \triangleq R_{(-\infty, 0]}$.

For any $u, v \in \mathcal{S}$ and any $\tau \in \mathbb{R}$, the $\tau$-concatenation of $u$ and $v$, denoted $u \wedge_{\tau} v$, is defined by: $\left(u \wedge_{\tau} v\right)(t)=u(t)$ if $t \leq \tau$; and $\left(u \wedge_{\tau} v\right)(t)=v(t)$ if $t>\tau$. We abbreviate $u \wedge v \triangleq u \wedge_{0} v$.

Define $\mathcal{V} \subseteq \mathcal{S}$ to be a signal space if and only if it is a vector space. Suppose additionally that $\mathcal{V}$ is a normed vector space and the norm $\|\cdot\|=\|\cdot\|_{\mathcal{V}}$ is also defined for signals of the form $T_{J} v, v \in \mathcal{V}, J \subseteq \mathbb{R}$. We can define a norm $\|\cdot\|_{J}$ on $\mathcal{S}_{J}$ by $\|v\|_{J}=\left\|T_{J} v\right\|$ for $v \in \mathcal{S}_{J}$ (define $\|v\|_{J} \triangleq \infty$ if $\left.T_{J} v \in \mathcal{S} \backslash \mathcal{V}\right)$. The extended space $\mathcal{V}_{e}$ of $\mathcal{V}$ is defined by

$$
\mathcal{V}_{e} \triangleq\left\{v \in \mathcal{S} \mid \forall a, b,(-\infty<a<b<\infty): T_{(a, b)} v \in \mathcal{V}\right\}
$$

and the interval space $\mathcal{V}(J) \triangleq R_{J} \mathcal{V}$ for any $J \subset \mathbb{R}$; we let $\mathcal{V}^{+}=R_{+} \mathcal{V}, \mathcal{V}^{-}=R_{-} \mathcal{V}, \mathcal{V}_{e}^{+}=R_{+} \mathcal{V}_{e}$ and $\mathcal{V}_{e}^{-}=R_{-} \mathcal{V}_{e}$

The essence of a system in the input-output sense is that only the relationship between inputs and outputs is relevant. In this sense, notions of a system should be made without the axiomatical postulation of state.

Definition 1: Given normed signal spaces $\mathcal{U}, \mathcal{Y}$ and $\mathcal{W} \triangleq$ $\mathcal{U} \times \mathcal{Y}$, a system $Q$ is defined to be a subset $\mathfrak{B}_{Q} \subset \mathcal{W}_{e}$. 
The signal pair $(u, y) \in \mathcal{U}_{e} \times \mathcal{Y}_{e}$ is called an input-output pair. At this stage, we do not impose any further requirements on the input/output partition. In the rest of this section, unless specified otherwise, we always regard $u \in \mathcal{U}_{e}$ as an input and $y \in \mathcal{Y}_{e}$ as an output for the system $Q$.

This is slightly different from both Zames's representation of input-output systems by operators [21] and Willems's structure of input-output systems by behaviours with input/output partition [15]. Here, we allow both $\left(u, y_{1}\right)$ and $\left(u, y_{2}\right)$ with $y_{1} \neq y_{2}$ belong to the same set $\mathfrak{B}_{Q}$. And it does not require that for any $u \in \mathcal{U}_{e}$ there exists a $y \in \mathcal{Y}_{e}$ such that $(u, y) \in \mathfrak{B}_{Q}$. For example, Let $\mathcal{U}=$ $\mathcal{Y} \triangleq L^{2}(\mathbb{R} ; \mathbb{R})$ and consider the system $Q$ represented by the set $\mathfrak{B}_{Q}=\left\{(u, y) \in \mathcal{U}_{e} \times \mathcal{Y}_{e} \mid y^{2}=u\right\}$. It is easy to verify that for $u(t)=e^{-2|t|}, t \in \mathbb{R}$ and $y(t)=e^{-|t|}, t \in \mathbb{R}$ we have both $(u, y)$ and $(u,-y)$ belong to $\mathfrak{B}_{Q}$, and that for $u(t)=-e^{-2|t|}, t \in \mathbb{R}$, there is no $y \in L_{e}^{2}(\mathbb{R} ; \mathbb{R})$ such that $(u, y) \in \mathfrak{B}_{Q}$. We will see in the subsequent sections that this definition of systems allow us to define initial conditions for systems appropriately and to treat in a unified manner systems with initial conditions of a structurally different type (e.g., both time delay distributed parameter and ODE systems), and to make it compatible with the definition of interconnected systems.

Definition 2: A system $Q$ (Definition 1) is said to be linear if the set $\mathfrak{B}_{Q}$ is a vector space, i.e., $\lambda_{1} w_{1}+\lambda_{2} w_{2} \in$ $\mathfrak{B}_{Q}$ for any $w_{1}, w_{2} \in \mathfrak{B}_{Q}$ and any $\lambda_{1}, \lambda_{2} \in \mathbb{R}$. It is said to be time-invariant if $w \in \mathfrak{B}_{Q}$ implies $w(\cdot+\tau) \in \mathfrak{B}_{Q}$ for all $\tau \in \mathbb{R}$.

Definition 3: Given normed signal spaces $\mathcal{U}$ and $\mathcal{Y}$, an operator $\Phi: \mathcal{U}_{e}^{+} \rightarrow \mathcal{Y}_{e}^{+}$is said to be causal if,

$$
\begin{array}{r}
\forall u, v \in \mathcal{U}_{e}^{+}, \forall t>0:\left[\left.u\right|_{[0, t]}=\left.v\right|_{[0, t]} \Rightarrow\right. \\
\left.\left.(\Phi u)\right|_{[0, t]}=\left.(\Phi v)\right|_{[0, t]}\right],
\end{array}
$$

while a system $Q$ (Definition 1) is said to be causal if

$$
\begin{array}{r}
\forall u, v \in \mathcal{U}_{e}, \forall t \in \mathbb{R}:\left[\left.u\right|_{(-\infty, t]}=\left.v\right|_{(-\infty, t]} \Rightarrow\right. \\
\left.\left.\mathfrak{B}_{Q}^{u}\right|_{(-\infty, t]}=\left.\mathfrak{B}_{Q}^{v}\right|_{(-\infty, t]}\right],
\end{array}
$$

where $\mathfrak{B}_{Q}^{u} \triangleq\left\{(u, y) \in \mathcal{W}_{e} \mid \exists y\right.$ such that $\left.(u, y) \in \mathfrak{B}_{Q}\right\}$.

The definition of a causal system generalises the definition of a casual operator. Note that any operator $\Phi: \mathcal{U}_{e}^{+} \rightarrow \mathcal{Y}_{e}^{+}$ can be represented by a system $\mathfrak{B}_{\Phi}=\left\{(u, y) \in \mathcal{U}_{e} \times\right.$ $\left.\mathcal{Y}_{e} \mid R_{-} y=R_{-} u=0, R_{+} y=\Phi\left(R_{+} u\right)\right\}$. According to above definition, the operator $\Phi$ is causal if and only if the system $\mathfrak{B}_{\Phi}$ is causal. In consideration of system's properties, we are interested in the trajectories on the positive direction time line $[t, \infty)$. In order to define the well-posedness of a system, we first introduce the two properties of existence and uniqueness of a system. In the following, we fix the initial time $t=0$ if not otherwise specified and use the notation $\mathfrak{B}_{Q}^{-}$ defined as follows to denote the system $Q$ 's past trajectories:

$$
\begin{aligned}
\mathfrak{B}_{Q}^{-} & \triangleq R_{-} \mathfrak{B}_{Q} \\
& =\left\{\begin{array}{l|c}
w_{-} \in \mathcal{W}_{e}^{-} & \begin{array}{c}
\exists w_{+} \in \mathcal{W}_{e}^{+} \text {such that } \\
w_{-} \wedge w_{+} \in \mathfrak{B}_{Q}
\end{array}
\end{array}\right\} .
\end{aligned}
$$

Definition 4: A system $Q$ (Definition 1) is said to have the existence property if for any $w_{-} \in \mathfrak{B}_{Q}^{-}$and any $u_{+} \in \mathcal{U}_{e}^{+}$ there exists a $y_{+} \in \mathcal{Y}_{e}^{+}$such that $w_{-} \wedge\left(u_{+}, y_{+}\right) \in \mathfrak{B}_{Q}$; and the uniqueness property if for any $w_{-} \in \mathfrak{B}_{Q}^{-}$and any $u_{+} \in \mathcal{U}_{e}^{+}$,

$$
\begin{array}{r}
w_{-} \wedge\left(u_{+}, y_{+}\right), w_{-} \wedge\left(u_{+}, \tilde{y}_{+}\right) \in \mathfrak{B}_{Q} \\
\text { with } y_{+}, \tilde{y}_{+} \in \mathcal{Y}_{e}^{+} \Rightarrow y_{+}=\tilde{y}_{+},
\end{array}
$$

and is well-posed if it has both the existence and uniqueness properties.

Well-posedness means that the future output $y_{+}$can be deduced from the set $\mathfrak{B}_{Q}$ (representing system properties) and the past input-output pair $\left(u_{-}, y_{-}\right)$and the future input $u_{+}$. We define the concept of output processes input by

$\left\{(u, y),\left(u, y^{\prime}\right) \in \mathfrak{B}_{Q}, y(t)=y^{\prime}(t)\right.$ for $\left.t \leq 0\right\} \Rightarrow\left\{y=y^{\prime}\right\}$.

Then well-posedness is equivalent to output processes input (see e.g. [20] in which the property of output processes input together with some other properties are postulated as axioms that need to be satisfied when defining input-output dynamical systems).

As discussed in intuitive terms in [22], the state is a classifier of input-output pasts. Thus intuitively the state should contain all the information of past history of the system which at any time together with the future input completely determine the future output. In the following, we will give a precise way to define the state of a system. The original idea is from $[4, \S 7]$ and from the viewpoint of observability, for any observable nonlinear system represented by a state space model, the initial state can be reconstructed from observed output signals given some known input signals (see e.g. [5]).

Given normed signal spaces $\mathcal{U}, \mathcal{Y}$ and $\mathcal{W} \triangleq \mathcal{U} \times \mathcal{Y}$, and consider the system $Q$ (Definition 1). We will now introduce an equivalence relation on $\mathfrak{B}_{Q}^{-} \triangleq R_{-} \mathfrak{B}_{Q}$ (see (1)) and show how this yields the state. Let $Q^{w_{-}}\left(u_{+}\right)$denote the set (possibly empty) of all future output trajectories generated by the system past input-output trajectories $w_{-} \in \mathfrak{B}_{Q}^{-}$and future input $u_{+} \in \mathcal{U}_{e}^{+}$, i.e.,

$$
Q^{w_{-}}\left(u_{+}\right) \triangleq\left\{y_{+} \in \mathcal{Y}_{e}^{+} \mid w_{-} \wedge\left(u_{+}, y_{+}\right) \in \mathfrak{B}_{Q}\right\} .
$$

Note that the set $Q^{w_{-}}\left(u_{+}\right)$is possibly empty for some $u_{+} \in$ $\mathcal{U}_{e}^{+}$. However, if the system $Q$ is well-posed, then there is a unique element in $Q^{w_{-}}\left(u_{+}\right)$for every $w_{-} \in \mathfrak{B}_{Q}^{-}$and every $u_{+} \in \mathcal{U}_{e}^{+}$. In this case, $Q^{w_{-}}(\cdot)$ defines an input-output operator from future inputs to future outputs.

Next we define an equivalence relation $\sim$ on $\mathfrak{B}_{Q}^{-} \triangleq$ $R_{-} \mathfrak{B}_{Q}$ (see (1)) by using (2) as follows: for any $w_{-}, \tilde{w}_{-} \in$ $\mathfrak{B}_{Q}^{-}$, we say

$$
w_{-} \sim \tilde{w}_{-} \Leftrightarrow Q^{w_{-}}\left(u_{+}\right)=Q^{\tilde{w}_{-}}\left(u_{+}\right), \forall u_{+} \in \mathcal{U}_{e}^{+} .
$$

Note that the definition of equivalence relation $\sim$ on $\mathfrak{B}_{Q}^{-}$ doesn't require the system $Q$ to be well-posed; if so then $Q^{w_{-}(\cdot)}$ defines an operator from $\mathcal{U}_{e}^{+}$to $\mathcal{Y}_{e}^{+}$. Given this equivalence relation $\sim$ on $\mathfrak{B}_{Q}^{-}$, the equivalence class of an 
element $w_{-}$in $\mathfrak{B}_{Q}^{-}$is the subset of all elements in $\mathfrak{B}_{Q}^{-}$which are equivalent to $w_{-}$denoted by $\left[w_{-}\right]$, defined as:

$$
\left[w_{-}\right] \triangleq\left\{\tilde{w}_{-} \in \mathfrak{B}_{Q}^{-} \mid \tilde{w}_{-} \sim w_{-}\right\} .
$$

Definition 5: We define $\mathfrak{S}_{Q}$ the initial state space of $Q$ at initial time 0 as the quotient set $\mathfrak{B}_{Q}^{-} / \sim$ which contains all equivalence classes in $\mathfrak{B}_{Q}^{-}$related to the equivalence relation $\sim$, i.e.,

$$
\mathfrak{S}_{Q} \triangleq \mathfrak{B}_{Q}^{-} / \sim \triangleq\left\{\left[w_{-}\right] \mid w_{-} \in \mathfrak{B}_{Q}^{-}\right\} .
$$

From the equivalence relation $\sim$, for any $x_{0} \in \mathfrak{S}_{Q}$, we can define the set $Q^{x_{0}}\left(u_{+}\right)$by:

$$
Q^{x_{0}}\left(u_{+}\right) \triangleq Q^{w_{-}}\left(u_{+}\right), \quad \forall u_{+} \in \mathcal{U}_{e}^{+}, \forall w_{-} \in x_{0} .
$$

Note that the above definition of initial state space doesn't require the system to be well-posed. If so, then, for every $w_{-} \in \mathfrak{B}_{Q}^{-}, Q^{w_{-}(\cdot)}$ is an operator from $\mathcal{U}_{e}^{+}$to $\mathcal{Y}_{e}^{+}$. This in turn implies that, for every $x_{0} \in \mathfrak{S}_{Q}, Q^{x_{0}}(\cdot)$ is an operator from $\mathcal{U}_{e}^{+}$to $\mathcal{Y}_{e}^{+}$.

If the initial time is chosen to be $t_{0} \in \mathbb{R}$ not 0 , we can similarly define the initial state space denoted by $\mathfrak{S}_{Q}^{t_{0}}$ of a system $Q$ at initial time $t_{0}$ by the same procedure.

We can use a real-valued function $\chi$ defined as follows to denote the size of elements in the initial state space $\mathfrak{S}_{Q}$ :

$$
\begin{aligned}
\chi: \quad \mathfrak{S}_{Q} & \rightarrow \mathbb{R}^{+}, \\
x_{0} & \mapsto \chi\left(x_{0}\right) \triangleq \inf \left\{\left\|w_{-}\right\| \mid w_{-} \in x_{0}\right\} .
\end{aligned}
$$

The function $\chi$ gives us information about the size of the smallest past input and output pair that can be used to generate the corresponding initial state. The computation of $\chi$ is a classical problem in optimal control theory (see e.g., [17] for state space models).

Definition 6: A function $\gamma:[0, a) \rightarrow \mathbb{R}_{+}$is said to be of class $\mathcal{K}$ if it is continuous, strictly increasing and satisfies $\gamma(0)=0$; moreover, if $a=\infty$ and $\lim _{s \rightarrow \infty} \gamma(s)=\infty$, then it is said to be of class $\mathcal{K}_{\infty}$. A function $\beta:[0, a) \times \mathbb{R}_{+} \rightarrow \mathbb{R}_{+}$ is said to be of class $\mathcal{K} \mathcal{L}$ if it is such that $\beta(\cdot, t) \in \mathcal{K}$ for each fixed $t \in \mathbb{R}_{+}$, and the function $\beta(s, \cdot)$ is decreasing and $\lim _{t \rightarrow \infty} \beta(s, t)=0$ for each fixed $s \in[0, a)$.

\section{GENERALISED NONLINEAR SMALL-GAIN THEOREM}

Given normed signal spaces $\mathcal{U}, \mathcal{Y}$ and $\mathcal{W} \triangleq \mathcal{U} \times \mathcal{Y}$. Consider the form of feedback configuration shown in Fig. 1. The signals $u_{i}$ and $y_{i}(i=0,1,2)$ belong to the extended signal spaces $\mathcal{U}_{e}$ and $\mathcal{Y}_{e}$, respectively. Define $w_{i}=\left(u_{i}, y_{i}\right)$ for $i=0,1,2$, thus $w_{i}$ for $i=0,1,2$ belong to $\mathcal{W}_{e}$. The symbols $G$ and $H$ represent two subsystems which consist of all the input-output signal pairs $w_{1}=\left(u_{1}, y_{1}\right) \in \mathcal{W}_{e}$ related to $G$ and all the output-input signal pairs $w_{2}=\left(u_{2}, y_{2}\right) \in$ $\mathcal{W}_{e}$ related to $H$, respectively, when the switches are open. (Here $G, H$ are relations (i.e., "multivalued functions").) When the switches are closed, the interconnection equation $w_{0}=w_{1}+w_{2}$ also holds.

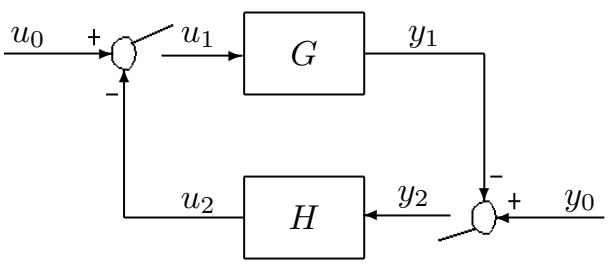

Fig. 1. Nonlinear feedback configuration $[G, H]$

The subsystems $G$ and $H$ are represented by the sets $\mathfrak{B}_{G}$ and $\mathfrak{B}_{H}$ (Definition 1) ${ }^{1}$, respectively; and the corresponding initial state spaces $\mathfrak{S}_{G}$ and $\mathfrak{S}_{H}$ at given initial time 0 are defined according to Definition 5. Note that the definitions of corresponding initial state spaces are not related to the well-posedness of the systems. We define the interconnected system $[G, H]$ shown in Fig. 1 (i.e., with the switches closed) by the following set $\mathfrak{B}_{[G, H]}$,

$$
\begin{aligned}
\mathfrak{B}_{[G, H]} \triangleq & \left\{\left(w_{0}, w_{1}, w_{2}\right) \in \mathcal{W}_{e} \times \mathcal{W}_{e} \times \mathcal{W}_{e} \mid\right. \\
& \left.w_{1} \in \mathfrak{B}_{G}, w_{2} \in \mathfrak{B}_{H}, w_{0}=w_{1}+w_{2}\right\} .
\end{aligned}
$$

In $\mathfrak{B}_{[G, H]}$ we view the external input $w_{0}$ as the (closed-loop) input and the internal signals $\left(w_{1}, w_{2}\right)$ as the (closed-loop) output.

We make the following notations to let the statement of the main result in this paper more concise. For any $x_{0} \in \mathfrak{S}_{G}$ and any $u_{1+} \in \mathcal{U}_{e}^{+}$, we let $G_{x_{0}} u_{1+}$ denote any of $y_{1+} \in$ $\mathcal{Y}_{e}^{+}$(if exists) such that $w_{1-} \wedge\left(u_{1+}, y_{1+}\right)$ (for any $w_{1-} \in$ $x_{0}$ ) is an input-output signal pair of $G$. Similarly, for any $z_{0} \in \mathfrak{S}_{H}$ and any $y_{2+} \in \mathcal{Y}_{e}^{+}$, we let $H_{z_{0}} y_{2+}$ denote any of $u_{2+} \in \mathcal{U}_{e}^{+}$(if exists) such that $w_{2-} \wedge\left(u_{2+}, y_{2+}\right)$ (for any $w_{2-} \in z_{0}$ ) is an output-input signal pair of $H$. Note that both $G_{x_{0}}$ and $H_{z_{0}}$ are "multivalued functions". Denote $\left[G_{x_{0}}, H_{z_{0}}\right]$ by the closed-loop relation which consists of all positive time input-output signal pairs $\left(w_{0+}, w_{1+}, w_{2+}\right)$ with $w_{0+} \in \mathcal{W}_{e}^{+}$denoting inputs and $\left(w_{1+}, w_{2+}\right) \in \mathcal{W}_{e}^{+} \times \mathcal{W}_{e}^{+}$ denoting outputs of $\left[G_{x_{0}}, H_{z_{0}}\right]$ such that

$$
\begin{gathered}
w_{0+}=w_{1+}+w_{2+}, \\
w_{1+} \triangleq\left(u_{1+}, G_{x_{0}} u_{1+}\right), \quad w_{2+} \triangleq\left(H_{z_{0}} y_{2+}, y_{2+}\right) .
\end{gathered}
$$

Lemma 1: Consider the feedback configuration shown in Fig. 1 (i.e., with the switches closed). Let $G, H$ be two causal time-invariant systems with above notations and $[G, H]$ be causal. Suppose that there are functions $\beta_{1}, \beta_{2} \in \mathcal{K} \mathcal{L}$ and $\gamma_{1}, \gamma_{2} \in \mathcal{K}_{\infty}$ such that for any $x_{0} \in \mathfrak{S}_{G}, z_{0} \in \mathfrak{S}_{H}$ and any $t>0, u_{1+} \in \mathcal{U}_{e}^{+}, y_{2+} \in \mathcal{Y}_{e}^{+}$,

$$
\begin{gathered}
\left|\left(G_{x_{0}} u_{1+}\right)(t)\right| \leq \beta_{1}\left(\chi\left(x_{0}\right), t\right)+\gamma_{1}\left(\left\|u_{1+}\right\|_{[0, t)}\right), \\
\left|\left(H_{z_{0}} y_{2+}\right)(t)\right| \leq \beta_{2}\left(\chi\left(z_{0}\right), t\right)+\gamma_{2}\left(\left\|y_{2+}\right\|_{[0, t)}\right),
\end{gathered}
$$

where (10) holds for all the "images" $G_{x_{0}} u_{1+}$ and $H_{z_{0}} y_{2+}$ of each $u_{1+} \in \mathcal{U}_{e}^{+}$and $y_{2+} \in \mathcal{Y}_{e}^{+}$, and the real-valued function $\chi$ is defined in (7). Then there are class $\mathcal{K}_{\infty}$ functions

\footnotetext{
${ }^{1}$ Note that when considering $H$, we need interchange the role of $\mathcal{U}_{e}$ and $\mathcal{Y}_{e}$ and think of $y_{2} \in \mathcal{Y}_{e}$ as the input and $u_{2} \in \mathcal{U}_{e}$ as the output.
} 
$\eta_{i}, \theta_{i},(i=1,2)$ independent of $x_{0}, z_{0}, u_{1+}, y_{2+}$ such that for any $t \geq 0$,

$$
\begin{aligned}
& \chi(x(t)) \leq \eta_{1}\left(\chi\left(x_{0}\right)\right)+\theta_{1}\left(\left\|\left(u_{1+}, G_{x_{0}} u_{1+}\right)\right\|_{[0, t)}\right), \\
& \chi(z(t)) \leq \eta_{2}\left(\chi\left(z_{0}\right)\right)+\theta_{2}\left(\left\|\left(H_{z_{0}} y_{2+}, y_{2+}\right)\right\|_{[0, t)}\right),
\end{aligned}
$$

where $x(t) \in \mathfrak{S}_{G}^{t}$ and $z(t) \in \mathfrak{S}_{H}^{t}$ are the corresponding states of $G$ and $H$ related to initial states $x_{0}$ and $z_{0}$ at time $t \geq 0$ with $x(0)=x_{0}$ and $z(0)=z_{0}$, respectively.

Proof: According to the definition of state in Definition 5, The inequalities (11) are immediately obtained by letting

$$
\eta_{i}(s)=\beta_{i}(s, 0)+s, \quad \theta_{i}(s)=\gamma_{i}(s)+s,
$$

for any $i=1,2$ and any $s \geq 0$.

Note that, for any function $\mu:[0, r) \rightarrow \mathbb{R}_{+}$of class $\mathcal{K}$, any function $\nu$ of class $\mathcal{K}_{\infty}$ and any $a \geq 0, b \geq 0$ with $a+b<r$, we have ${ }^{2}$

$$
\mu(a+b) \leq \mu \circ(I+\nu)(a)+\mu \circ\left(I+\nu^{-1}\right)(b) .
$$

where $I$ stands for the identity function, i.e., $I(s)=s$ for any $s \geq 0$.

Theorem 1: Under the same conditions and notations in Lemma 1. If there exist two functions $\rho \in \mathcal{K}_{\infty}$ and $\varepsilon \in \mathcal{K}_{\infty}$ such that

$$
\gamma_{1} \circ(I+\rho) \circ \gamma_{2}(s) \leq(I+\varepsilon)^{-1}(s), \quad \forall s \geq 0,
$$

Then, for any function $\alpha \in \mathcal{K}_{\infty}$, there exists a function $\beta \in$ $\mathcal{K} \mathcal{L}$ such that for any $i=1,2$ and all $t>0$, and all $w_{0+} \in$ $\mathcal{U}_{e}^{+} \times \mathcal{Y}_{e}^{+}$,

$$
\left|w_{i+}(t)\right| \leq \beta\left(\chi\left(x_{0}, z_{0}\right), t\right)+(\alpha+\gamma)\left(\left\|w_{0+}\right\|_{[0, t)}\right),
$$

where the real-valued function $\chi$ is defined in (7) and $\gamma \in$ $\mathcal{K}_{\infty}$ is defined as follows, for any $r \geq 0$,

$$
\left\{\begin{aligned}
\gamma(r) & =\left(I+\left(I+\rho^{-1}\right)^{2} \circ \gamma_{3}+\left(I+\varepsilon^{-1}\right)^{2} \circ \gamma_{4}\right)(r), \\
\gamma_{3}(r) & =\left(I+\gamma_{2} \circ\left(I+\varepsilon^{-1}\right)^{2}\right)(r), \\
\gamma_{4}(r) & =\left(I+\gamma_{1} \circ\left(I+\rho^{-1}\right)^{2}\right)(r) .
\end{aligned}\right.
$$

Proof: Choose $s=(I+\varepsilon) \circ \gamma_{1}(\hat{s}),(\hat{s} \geq 0)$ in (13), we have $\gamma_{1} \circ(I+\rho) \circ \gamma_{2} \circ(I+\varepsilon) \circ \gamma_{1}(\hat{s}) \leq \gamma_{1}(\hat{s}),(\hat{s} \geq 0)$. Hence, we get

$$
\gamma_{2} \circ(I+\varepsilon) \circ \gamma_{1}(\hat{s}) \leq(I+\rho)^{-1}(\hat{s}), \quad \forall \hat{s} \geq 0,
$$

For any initial states $x_{0} \in \mathfrak{S}_{G}$ and $z_{0} \in \mathfrak{S}_{H}$ and any $w_{0+}=\left(u_{0+}, y_{0+}\right) \in \mathcal{U}^{+} \times \mathcal{Y}^{+}$, we define two nonnegative constants $b_{10}=\beta_{1}\left(\chi\left(x_{0}\right), 0\right)$ and $b_{20}=\beta_{2}\left(\chi\left(z_{0}\right), 0\right)$. Then, from (9) and (10), we obtain that

$$
\begin{aligned}
\left\|u_{1+}\right\|_{[0, t)} & \leq\left\|u_{0+}\right\|_{[0, t)}+\left\|H_{z_{0}} y_{2+}\right\|_{[0, t)} \\
& \leq\left\|u_{0+}\right\|_{[0, t)}+b_{20}+\gamma_{2}\left(\left\|y_{2+}\right\|_{[0, t)}\right), \forall t>0
\end{aligned}
$$

Similarly, we have

$$
\begin{aligned}
\left\|y_{2+}\right\|_{[0, t)} & \leq\left\|y_{0+}\right\|_{[0, t)}+\left\|G_{x_{0}} u_{1+}\right\|_{[0, t)} \\
& \leq\left\|y_{0+}\right\|_{[0, t)}+b_{10}+\gamma_{1}\left(\left\|u_{1+}\right\|_{[0, t)}\right), \forall t>0
\end{aligned}
$$

${ }^{2}$ if $b \leq \nu(a)$ then $\mu(a+b) \leq \mu \circ(I+\nu)(a)$; and if $a \leq \nu^{-1}(b)$ then $\mu(a+b) \leq \mu \circ\left(I+\nu^{-1}\right)(b)$.
Hence, we get

$$
\begin{aligned}
\left\|u_{1+}\right\|_{[0, t)} \leq & \left\|u_{0+}\right\|_{[0, t)}+b_{20} \\
& +\gamma_{2} \circ(I+\varepsilon) \circ \gamma_{1}\left(\left\|u_{1+}\right\|_{[0, t)}\right) \\
& +\gamma_{2} \circ\left(I+\varepsilon^{-1}\right)\left(\left\|y_{0+}\right\|_{[0, t)}+b_{10}\right) .
\end{aligned}
$$

From (16), (17) and $\left(I-(I+\rho)^{-1}\right)^{-1}(\cdot)=\left(I+\rho^{-1}\right)(\cdot)$, we have, for all $t>0$,

$$
\begin{aligned}
\left\|u_{1+}\right\|_{[0, t)} \leq & \left(I+\rho^{-1}\right)\left(\left\|u_{0+}\right\|_{[0, t)}+b_{20}\right. \\
& \left.+\gamma_{2} \circ\left(I+\varepsilon^{-1}\right)\left(\left\|y_{0+}\right\|_{[0, t)}+b_{10}\right)\right) .
\end{aligned}
$$

Similarly, we have, for all $t>0$,

$$
\begin{aligned}
\left\|y_{2+}\right\|_{[0, t)} \leq & \left(I+\varepsilon^{-1}\right)\left(\left\|y_{0+}\right\|_{[0, t)}+b_{10}\right. \\
& \left.+\gamma_{1} \circ\left(I+\rho^{-1}\right)\left(\left\|u_{0+}\right\|_{[0, t)}+b_{20}\right)\right) .
\end{aligned}
$$

Note that, for all $t>0,\left\|u_{2+}\right\|_{[0, t)} \leq\left\|u_{0+}\right\|_{[0, t)}+\left\|u_{1+}\right\|_{[0, t)}$ and $\left\|y_{1+}\right\|_{[0, t)} \leq\left\|y_{0+}\right\|_{[0, t)}+\left\|y_{2+}\right\|_{[0, t)}$. Hence, by applying (12) to (18) and (19), we obtain that there exist a class $\mathcal{K}_{\infty}$ function $\kappa$ such that, for any $i=1,2$ and all $t>0$,

$$
\left\|w_{i+}\right\|_{[0, t)} \leq \gamma\left(\left\|w_{0+}\right\|_{[0, t)}\right)+\kappa\left(\chi\left(x_{0}, z_{0}\right)\right),
$$

where $\gamma \in \mathcal{K}_{\infty}$ is defined in (15).

From (11) in Lemma 1 and (20), and by using (12), we know that, for any $t>0$,

$$
\begin{aligned}
\chi(x(t), z(t)) \leq & \left(\eta_{1}+\eta_{2}\right)\left(\chi\left(x_{0}, z_{0}\right)\right) \\
& +\left(\theta_{1}+\theta_{2}\right)\left(\max \left\{\left\|w_{1+}\right\|_{[0, t)},\left\|w_{2+}\right\|_{[0, t)}\right\}\right) \\
\leq & \delta_{1}\left(\chi\left(x_{0}, z_{0}\right)\right)+\delta_{2}\left(\left\|w_{0+}\right\|_{[0, \infty)}\right) \\
\triangleq & s_{\infty}, \quad \forall t>0,
\end{aligned}
$$

where $x(t)$ and $z(t)$ are the corresponding states at time $t>$ 0 of $G$ and $H$ related to initial states $x_{0}$ and $z_{0}$, respectively; and $\delta_{1}(s)=\left(\eta_{1}+\eta_{2}\right)(s)+\left(\theta_{1}+\theta_{2}\right) \circ\left(I+\rho^{-1}\right) \circ \kappa(s)$ and $\delta_{2}(s)=\left(\theta_{1}+\theta_{2}\right) \circ(I+\rho) \circ \gamma(s), \forall s \geq 0$.

It's easy to see that both $\delta_{1}$ and $\delta_{2}$ are of class $\mathcal{K}_{\infty}$ functions. Next we estimate the bound of $\left|w_{i}(t)\right|, i=1,2$ for any $t>0$. Since both $G$ and $H$ are causal and timeinvariant, by using (10) and (21), we have for any $t>0$ and any $u_{1+} \in \mathcal{U}_{e}^{+}$, and any $y_{2+} \in \mathcal{Y}_{e}^{+}$,

$$
\begin{aligned}
\left|\left(G_{x_{0}} u_{1+}\right)(t)\right| & \leq \beta_{1}(\chi(x(t / 2)), t / 2)+\gamma_{1}\left(\left\|u_{1+}\right\|_{\left[\frac{t}{2}, t\right)}\right) \\
& \leq \beta_{1}\left(s_{\infty}, t / 2\right)+\gamma_{1}\left(\left\|u_{1+}\right\|_{\left[\frac{t}{2}, t\right)}\right), \\
\left|\left(H_{z_{0}} y_{2+}\right)(t)\right| & \leq \beta_{2}(\chi(z(t / 2)), t / 2)+\gamma_{2}\left(\left\|y_{2+}\right\|_{\left[\frac{t}{2}, t\right)}\right) \\
& \leq \beta_{2}\left(s_{\infty}, t / 2\right)+\gamma_{2}\left(\left\|y_{2+}\right\|_{\left[\frac{t}{2}, t\right)}\right) .
\end{aligned}
$$

Thus, by applying (9) and (22), we have, for all $t>0$,

$$
\begin{aligned}
\left|u_{1+}(t)\right| & \leq\left|u_{0+}(t)\right|+\left|\left(H_{z_{0}} y_{2+}\right)(t)\right| \\
& \leq\left\|u_{0+}\right\|_{[0, t)}+\beta_{2}\left(s_{\infty}, t / 2\right)+\gamma_{2}\left(\left\|y_{2+}\right\|_{\left[\frac{t}{2}, t\right)}\right) \\
\left|y_{2+}(t)\right| & \leq\left|y_{0+}(t)\right|+\left\|\left(G_{x_{0}} u_{1+}\right)(t)\right\| \\
& \leq\left\|y_{0+}\right\|_{[0, t)}+\beta_{1}\left(s_{\infty}, t / 2\right)+\gamma_{1}\left(\left\|u_{1+}\right\|_{\left[\frac{t}{2}, t\right)}\right) .
\end{aligned}
$$


Hence, we get, for all $t>0$,

$$
\begin{aligned}
\left|u_{1+}(t)\right| \leq & \left\|u_{0+}\right\|_{[0, t)}+\beta_{2}\left(s_{\infty}, t / 2\right) \\
& +\gamma_{2} \circ(I+\varepsilon) \circ \gamma_{1}\left(\left\|u_{1+}\right\|_{\left[\frac{t}{2}, t\right)}\right) \\
& +\gamma_{2} \circ\left(I+\varepsilon^{-1}\right)\left(\left\|y_{0+}\right\|_{[0, t)}+\beta_{1}\left(s_{\infty}, t / 2\right)\right) \\
\leq & \left\|u_{0+}\right\|_{[0, t)}+\beta_{2}\left(s_{\infty}, t / 2\right) \\
& +(I+\rho)^{-1}\left(\left\|u_{1+}\right\|_{\left[\frac{t}{2}, t\right)}\right) \\
& +\gamma_{2} \circ\left(I+\varepsilon^{-1}\right)\left(\left\|y_{0+}\right\|_{[0, t)}+\beta_{1}\left(s_{\infty}, t / 2\right)\right) \\
\leq & \beta_{3}\left(s_{\infty}, t\right)+(I+\rho)^{-1}\left(\left\|u_{1+}\right\|_{\left[\frac{t}{2}, t\right)}\right) \\
& +\gamma_{3}\left(\left\|w_{0+}\right\|_{[0, t)}\right)
\end{aligned}
$$

with $\gamma_{3} \in \mathcal{K}_{\infty}$ defined in (15) and $\beta_{3} \in \mathcal{K} \mathcal{L}$ defined by

$$
\begin{aligned}
\beta_{3}(r, s) \triangleq & \beta_{2}(r, s / 2)+\gamma_{2} \circ\left(I+\varepsilon^{-1}\right) \\
& \left.\circ(I+\varepsilon) \circ \beta_{1}(r, s / 2)\right), \forall r \geq 0, \forall s \geq 0 .
\end{aligned}
$$

Next we apply $[9, \text { Lemma A.1 }]^{3}$ to (23), it follows that a function $\beta_{4}$ of class $\mathcal{K} \mathcal{L}$ exists such that, for all $t>0$,

$$
\begin{aligned}
\left|u_{1+}(t)\right| \leq & \beta_{4}\left(s_{\infty}, t\right)+\left(I-(I+\rho)^{-1}\right)^{-1} \\
& \circ\left(I+\rho^{-1}\right) \circ \gamma_{3}\left(\left\|w_{0+}\right\|_{[0, \infty)}\right) \\
= & \beta_{4}\left(s_{\infty}, t\right)+\left(I+\rho^{-1}\right)^{2} \circ \gamma_{3}\left(\left\|w_{0+}\right\|_{[0, \infty)}\right),
\end{aligned}
$$

where we use the fact that $\left(I-(I+\rho)^{-1}\right)^{-1}(s)=(I+$ $\left.\rho^{-1}\right)(s)$ for any $s \geq 0$.

Similarly, there exist a function $\beta_{5} \in \mathcal{K} \mathcal{L}$ such that, for all $t>0$,

$$
\begin{aligned}
\left|y_{2+}(t)\right| \leq & \beta_{5}\left(s_{\infty}, t\right) \\
& +\left(I+\varepsilon^{-1}\right)^{2} \circ \gamma_{4}\left(\left\|w_{0+}\right\|_{[0, \infty)}\right)
\end{aligned}
$$

with $\gamma_{4} \in \mathcal{K}_{\infty}$ defined in (15).

Note that, for all $t>0,\left|u_{2+}(t)\right| \leq\left|u_{0+}(t)\right|+\left|u_{1+}(t)\right|$ and $\left|y_{1+}(t)\right| \leq\left|y_{0+}(t)\right|+\left|y_{2+}(t)\right|$. Hence, from (24) and (25), we have, for all $t>0$,

$$
\left|w_{i+}(t)\right| \leq \beta_{6}\left(s_{\infty}, t\right)+\gamma\left(\left\|w_{0+}\right\|_{[0, \infty)}\right), \quad i=1,2,
$$

with $\beta_{6}(r, s) \triangleq \max \left\{\beta_{4}(r, s), \beta_{5}(r, s)\right\}, \forall r \geq 0, \forall s \geq 0$, and $\gamma \in \mathcal{K}_{\infty}$ defined in (15).

Since $s_{\infty}=\delta_{1}\left(\chi\left(x_{0}, z_{0}\right)\right)+\delta_{2}\left(\left\|w_{0+}\right\|_{[0, \infty)}\right)$ (see (21)), from (20) and (26), we have for any $t \geq 0$,

$$
\begin{aligned}
\left|w_{i+}(t)\right| \leq & \gamma\left(\left\|w_{0+}\right\|_{[0, \infty)}\right)+\min \left\{\kappa\left(\chi\left(x_{0}, z_{0}\right)\right),\right. \\
& \left.\beta_{6}\left(\delta_{1}\left(\chi\left(x_{0}, z_{0}\right)\right)+\delta_{2}\left(\left\|w_{0+}\right\|_{[0, \infty)}\right), t\right)\right\} .
\end{aligned}
$$

Given any function $\alpha$ of $\mathcal{K}_{\infty}$, there are only two cases $\chi\left(x_{0}, z_{0}\right) \leq \kappa^{-1} \circ \alpha\left(\left\|w_{0+}\right\|_{[0, \infty)}\right)$ or $\left\|w_{0+}\right\|_{[0, \infty)} \leq \alpha^{-1} \circ$ $\kappa\left(\chi\left(x_{0}, z_{0}\right)\right)$, thus from (27) and by considering the fact that

${ }^{3}$ Let $\beta \in \mathcal{K} \mathcal{L}, \lambda \in \mathcal{K}_{\infty}$ such that $I-\lambda \in \mathcal{K}_{\infty}$, and let $\mu \in(0,1]$. Then, for any function $\delta$ such that $\delta-I \in \mathcal{K}_{\infty}$, a function $\hat{\beta} \in \mathcal{K} \mathcal{L}$ exists such that, for any $s \geq 0, d \geq 0$ and any nonnegative real function $z(t)$, defined and essentially bounded on $[0, \infty)$ and satisfying $z(t) \leq$ $\beta(s, t)+\lambda\left(\|z\|_{[\mu t, \infty)}\right)+d$ for any $t \in[0,+\infty)$, we have $z(t) \leq \hat{\beta}(s, t)+$ $(I-\lambda)^{-1} \circ \delta(d)$ for any $t \in[0,+\infty)$. [Here, when applying to (23), we let $\beta \triangleq \beta_{3}, \lambda \triangleq(I+\rho)^{-1}, \mu \triangleq \frac{1}{2}, \delta \triangleq I+\rho^{-1}$ and $\left.d=\gamma_{3}\left(\left\|w_{0+}\right\|_{[0, \infty)}\right).\right]$ for any fixed $t>0$ the function $\beta_{6}(\cdot, t) \in \mathcal{K}$, we have for any $t \geq 0$,

$$
\begin{aligned}
\left|w_{i+}(t)\right| \leq & \gamma\left(\left\|w_{0+}\right\|_{[0, \infty)}\right)+\kappa \circ \kappa^{-1} \circ \alpha\left(\left\|w_{0+}\right\|_{[0, \infty)}\right) \\
& +\beta_{6}\left(\delta_{1}\left(\chi\left(x_{0}, z_{0}\right)\right)+\delta_{2} \circ \alpha^{-1} \circ \kappa\left(\chi\left(x_{0}, z_{0}\right)\right), t\right) .
\end{aligned}
$$

Thus, by the causality of $[G, H]$ and the definition of extended space, for any $\alpha \in \mathcal{K}_{\infty}$ and any $i=1,2$ and all $t>0$, and all $w_{0+} \in \mathcal{U}_{e}^{+} \times \mathcal{Y}_{e}^{+}$, we have,

$$
\left|w_{i+}(t)\right| \leq \beta\left(\chi\left(x_{0}, z_{0}\right), t\right)+(\alpha+\gamma)\left(\left\|w_{0+}\right\|_{[0, t)}\right),
$$

with $\beta(r, s) \triangleq \beta_{6}\left(\left(\delta_{1}+\delta_{2} \circ \alpha^{-1} \circ \kappa\right)(r), s\right), \forall r \geq 0, \forall s \geq 0$, and $\gamma \in \mathcal{K}_{\infty}$ defined in (15).

\section{A. Illustration}

We next illustrate Theorem 1 by considering the following special example for systems with time delay and nonzero initial conditions. Consider the feedback configuration shown in Fig. 1. The subsystem $G$ is defined by the set

$$
\begin{gathered}
\mathfrak{B}_{G}=\left\{w_{1} \in \mathcal{W}_{e} \mid w_{1}=\left(u_{1}, y_{1}\right) \text { satisfies }(29)\right\}, \\
\dot{y}_{1}(t)=-a y_{1}\left(t-\tau_{1}\right)+\varepsilon\left(e^{u_{1}(t)}-1\right),
\end{gathered}
$$

and the subsystem $H$ is defined by the set

$$
\begin{gathered}
\mathfrak{B}_{H}=\left\{w_{2} \in \mathcal{W}_{e} \mid w_{2}=\left(u_{2}, y_{2}\right) \text { satisfies }(31)\right\}, \\
\dot{u}_{2}(t)=\operatorname{sat}\left\{-b u_{2}\left(t-\tau_{2}\right)+\operatorname{sat}\left[y_{2}(t)\right]\right\},
\end{gathered}
$$

with the interconnection conditions $u_{0}=u_{1}+u_{2}$ and $y_{0}=$ $y_{1}+y_{2}$, where $a>0, b>0$ are fixed real numbers, and $\varepsilon \in$ $\mathbb{R}, \tau_{1}>0, \tau_{2}>0$ are small parameters, and the saturation function sat $: \mathbb{R} \rightarrow \mathbb{R}$ satisfying $\operatorname{sat}(s)=s$ when $|s| \leq 1$ and $\operatorname{sat}(s)=1$ when $s>1$ and $\operatorname{sat}(s)=-1$ when $s<-1$.

The corresponding initial state spaces $\mathfrak{S}_{G}$ and $\mathfrak{S}_{H}$ at given initial time 0 are defined according to Definition 5 . The interconnected system $[G, H]$ is defined as (8). Both $G$ and $H$ are causal and time-invariant, and $[G, H]$ is causal.

Note that, for any $\kappa_{1}>0$ and any $\varepsilon_{1} \in(0, a)$, when $\dot{x}(t)=-a x\left(t-\tau_{1}\right)+f(t)$, the following inequality

$$
\begin{aligned}
|x(t)| \geq \max \left\{\begin{array}{l}
\frac{\left(1+\kappa_{1}\right) a^{2} \tau_{1}}{a-\varepsilon_{1}}\|x\|_{\left[t-2 \tau_{1}, t\right]}, \\
\frac{\left(1+1 / \kappa_{1}\right)\left(a \tau_{1}+1\right)}{a-\varepsilon_{1}}\|f\|_{\left[t-\tau_{1}, t\right]}
\end{array}\right\}
\end{aligned}
$$

implies ${ }^{4}$ that $\frac{d}{d t} x^{2}(t) \leq-2 \varepsilon_{1}|x(t)|^{2}$. Also note that, for any $\kappa_{2}>0$ and any $\varepsilon_{2} \in(0, b)$, when $\dot{z}(t)=\operatorname{sat}\left[-b z\left(t-\tau_{2}\right)+\right.$ $g(t)]$, the following inequality

$$
\begin{aligned}
& |z(t)| \geq \max \left\{\frac{\left(1+\kappa_{2}\right) b^{2} \tau_{2}}{b-\varepsilon_{2}}\|z\|_{\left[t-2 \tau_{2}, t\right]},\right. \\
& \left.\frac{\left(1+1 / \kappa_{2}\right)\left(b \tau_{2}+1\right)}{b-\varepsilon_{2}}\|g\|_{\left[t-\tau_{2}, t\right]}\right\}
\end{aligned}
$$

${ }^{4}$ This follows from $\dot{x}(t)=-a x(t)+a x(t)-a x\left(t-\tau_{1}\right)+f(t)=$ $-a x(t)+a \tau_{1} \dot{x}\left(\theta_{1}\right)+f(t)$ for some $\theta_{1} \in\left(t-\tau_{1}, t\right)$ that $|\dot{x}(t)+a x(t)| \leq$ $a^{2} \tau_{1}\|x\|_{\left[t-2 \tau_{1}, t\right]}+\left(a \tau_{1}+1\right)\|f\|_{\left[t-\tau_{1}, t\right]}$. By using the fact that $A+$ $B \leq \max \left\{\left(1+\kappa_{1}\right) A,\left(1+1 / \kappa_{1}\right) B\right\}$ for any $A \geq 0, B \geq 0$ and $\kappa_{1}>0$ in the previous inequality, we have $|\dot{x}(\bar{t})+a x(t)| \leq$ $\max \left\{\left(1+\kappa_{1}\right) a^{2} \tau_{1}\|x\|_{\left[t-2 \tau_{1}, t\right]},\left(1+1 / \kappa_{1}\right)\left(a \tau_{1}+1\right)\|f\|_{\left[t-\tau_{1}, t\right]}\right\} \leq$ $\left(a-\varepsilon_{1}\right)|x(t)|$ and thus $x(t) \dot{x}(t) \leq-\varepsilon_{1}|x(t)|^{2}$. 
implies ${ }^{5}$ that $\frac{d}{d t} z^{2}(t) \leq-2|z(t)| \operatorname{sat}\left(\varepsilon_{2}|z(t)|\right)$.

So, for the subsystems $G$ and $H$, by applying the Razumikhin-type theorem (see [19, Theorem 2]), we have that, for any $\kappa_{1}>0, \kappa_{2}>0$ and any $\varepsilon_{1} \in(0, a)$, $\varepsilon_{2} \in(0, b)$, if $\frac{\left(1+\kappa_{1}\right) a^{2} \tau_{1}}{a-\varepsilon_{1}}<1$ and $\frac{\left(1+\kappa_{2}\right) b^{2} \tau_{2}}{b-\varepsilon_{2}}<1$, then there exist $\beta_{\kappa_{1}, \varepsilon_{1}} \stackrel{a-\varepsilon_{1}}{\in} \mathcal{K} \mathcal{L}, \beta_{\kappa_{2}, \varepsilon_{2}} \in \mathcal{K} \mathcal{L}$ such that, for any $x_{0} \triangleq\left[\left(u_{1-}, y_{1-}\right)\right] \in \mathfrak{S}_{G}, z_{0} \triangleq\left[\left(u_{2-}, y_{2-}\right)\right] \in \mathfrak{S}_{H}$, and any $u_{1+} \in \mathcal{U}_{e}^{+}, y_{2+} \in \mathcal{Y}_{e}^{+}$, and any $t>0$,

$$
\begin{aligned}
\left|y_{1+}(t)\right| & \leq \beta_{\kappa_{1}, \varepsilon_{1}}\left(\left\|y_{1-}\right\|_{\left[-2 \tau_{1}, 0\right]}, t\right)+\gamma_{1}\left(\left\|u_{1+}\right\|_{[0, t)}\right) \\
& \leq \beta_{\kappa_{1}, \varepsilon_{1}}\left(\chi\left(x_{0}\right), t\right)+\gamma_{1}\left(\left\|u_{1+}\right\|_{[0, t)}\right), \\
\left|u_{2+}(t)\right| & \leq \beta_{\kappa_{2}, \varepsilon_{2}}\left(\left\|u_{2-}\right\|_{\left[-2 \tau_{2}, 0\right]}, t\right)+\gamma_{2}\left(\left\|y_{2+}\right\|_{[0, t)}\right) \\
& \leq \beta_{\kappa_{2}, \varepsilon_{2}}\left(\chi\left(z_{0}\right), t\right)+\gamma_{2}\left(\left\|y_{2+}\right\|_{[0, t)}\right),
\end{aligned}
$$

with the real-valued function $\chi$ defined in (7) and $\gamma_{1} \in \mathcal{K}_{\infty}$, $\gamma_{2} \in \mathcal{K}_{\infty}$ defined as follows

$$
\begin{aligned}
& \gamma_{1}(s)=\frac{\left(1+1 / \kappa_{1}\right)\left(a \tau_{1}+1\right)}{a-\varepsilon_{1}}|\varepsilon|\left(e^{s}-1\right), \quad \forall s \geq 0, \\
& \gamma_{2}(s)=\frac{\left(1+1 / \kappa_{2}\right)\left(b \tau_{2}+1\right)}{b-\varepsilon_{2}} \operatorname{sat}(s), \quad \forall s \geq 0 .
\end{aligned}
$$

Theorem 1 now asserts that, for the interconnected system $[G, H]$, the inequalities (14) will hold if there exist two functions $\rho_{1}(s), \rho_{2}(s), s \geq 0$ of class $\mathcal{K}_{\infty}$ such that

$$
\gamma_{1} \circ\left(I+\rho_{1}\right) \circ \gamma_{2}(s) \leq\left(I+\rho_{2}\right)^{-1}(s), \quad \forall s \geq 0 .
$$

Graphically, the above inequality (32) is equivalent to say that the distance between the curves $\left(x, \gamma_{2}(x)\right)$ and $\left(\gamma_{1}(y), y\right)$ grows without bound in the first quadrant of Cartesian coordinate system $(x, y)$. So, if $\gamma_{1} \circ \gamma_{2}(1)<1$, then (32) will be satisfied for some functions $\rho_{1}, \rho_{2}$ of class $\mathcal{K}_{\infty}$.

Hence, for the interconnected system $[G, H]$, the inequalities (14) will hold if the parameters $\varepsilon \in \mathbb{R}, \tau_{1}>0, \tau_{2}>0$ satisfying

$$
\left\{\begin{array}{l}
\tau_{1}<\tau_{1}^{*} \triangleq \frac{a-\varepsilon_{1}}{\left(1+\kappa_{1}\right) a^{2}}, \quad \tau_{2}<\tau_{2}^{*} \triangleq \frac{b-\varepsilon_{2}}{\left(1+\kappa_{2}\right) b^{2}}, \\
|\varepsilon|<\frac{a-\varepsilon_{1}}{\left(1+\frac{1}{\kappa_{1}}\right)\left(a \tau_{1}+1\right)\left\{\exp \left[\frac{\left(1+1 / \kappa_{2}\right)\left(b \tau_{2}+1\right)}{b-\varepsilon_{2}}\right]-1\right\}},
\end{array}\right.
$$

for any $\kappa_{1}>0, \kappa_{2}>0$ and any $\varepsilon_{1} \in(0, a), \varepsilon_{2} \in(0, b)$. Note that for any $\tau_{1}^{*}<1 / a$ and any $\tau_{2}^{*}<1 / b$, we can always choose $\kappa_{1}, \kappa_{2}$ and $\varepsilon_{1}, \varepsilon_{2}$ so that the above inequalities are satisfied.

\section{CONCLUSION}

In this paper, we develop a general ISS-type nonlinear small-gain theorem by defining a system and its corresponding initial conditions from an input-output point of view. It inherits the property of classical small-gain theorem that the question of stability is absolutely disconnected from the question of existence and uniqueness. An illustration of our

\footnotetext{
${ }^{5}$ Similarly, this follows from $\dot{z}(t)=$ sat $\left(-b z(t)+b \tau_{2} \dot{z}\left(\theta_{2}\right)+g(t)\right)$ for some $\theta_{2} \in\left(t-\tau_{2}, t\right)$ and from $\left|b \tau_{2} \dot{z}\left(\theta_{2}\right)+g(t)\right| \leq b^{2} \tau_{2}\|z\|_{\left[t-2 \tau_{2}, t\right]}+$ $\left(b \tau_{2}+1\right)\|g\|_{\left[t-\tau_{2}, t\right]} \leq \max \left\{\left(1+\kappa_{2}\right) b^{2} \tau_{2}\|z\|_{\left[t-2 \tau_{2}, t\right]},(1+\right.$ $\left.\left.1 / \kappa_{2}\right)\left(b \tau_{2}+1\right)\|g\|_{\left[t-\tau_{2}, t\right]}\right\} \leq\left(b-\varepsilon_{2}\right)|z(t)|$ that $z(t) \dot{z}(t) \leq z(t)$ sat $(-$ $\left.b z(t)+\left(b-\varepsilon_{2}\right) z(t)\right)=-|z(t)| \operatorname{sat}\left(\varepsilon_{2}|z(t)|\right)$.
}

main result is provided. On the one hand this can be viewed as an extension of the operator theoretical input-output theory to include initial conditions, but retaining the generality of the system class, and on the other hand an extension of the ISS/IOS framework to incorporate very general system classes (e.g., not tied to state space representations).

\section{REFERENCES}

[1] Z. Chen and J. Huang, A simplified small gain theorem for timevarying nonlinear systems, IEEE Trans. on Automatic Control, 50 (2005), pp. 1904-1908.

[2] S. Dashkovskiy, B. S. Rüffer, and F. R. Wirth, An ISS small gain theorem for general networks, Mathematics of Control, Signals, and Systems, 19 (2007), pp. 93-122.

[3] C. A. Desoer and M. Vidyasagar, Feedback systems: input-output properties, Academic Press (New York), 1975.

[4] M. French and M. Mueller, Nonlinear high gain separation principles and fast sampling results ensuring robust stability. Submitted for publication.

[5] J. P. Gauthier and I. Kupka, Deterministic observation theory and applications, Cambridge University Press, 2001.

[6] D. J. Hill, A generalization of the small-gain theorem for nonlinear feedback systems, Automatica, 27 (1991), pp. 1043-1045.

[7] Z.-P. Jiang and I. M. Y. Marcels, A small-gain control method for nonlinear cascaded systems with dynamic uncertainties, IEEE Trans. on Automatic Control, 42 (1997), pp. 292-308.

[8] Z.-P. Jiang, I. M. Y. Mareels, and Y. Wang, A lyapunov formulation of the nonlinear small-gain theorem for interconnected ISS systems, Automatica, 32 (1996), pp. 1211-1215.

[9] Z.-P. Jiang, A. R. Teel, and L. Praly, Small-gain theorem for ISS systems and applications, Mathematics of Control, Signals, and Systems (MCSS), 7 (1994), pp. 95-120.

[10] Z.-P. Jiang and Y. Wang, A generalization of the nonlinear small-gain theorem for large-scale complex systems, in Intelligent Control and Automation, 2008. WCICA 2008. 7th World Congress on, june 2008, pp. $1188-1193$

[11] D. Liberzon and D. Nešić, Stability analysis of hybrid systems via small-gain theorems, in Hybrid Systems: Computation and Control, vol. 3927 of Lecture Notes in Computer Science, Springer Berlin Heidelberg, 2006, pp. 421-435.

[12] J. Lu and R. E. Skelton, Mean-square small gain theorem for stochastic control: discrete-time case, IEEE Trans. on Automatic Control, 47 (2002), pp. 490-494.

[13] I. M. Y. Mareels and D. J. Hill, Monotone stability of nonlinear feedback systems, J. Math. Syst. Estim. Control, 2 (1992), pp. 275-291.

[14] D. Nešić and A. R. Teel, A lyapunov-based small-gain theorem for hybrid ISS systems, in Decision and Control, 2008. CDC 2008. 47th IEEE Conference on, dec. 2008, pp. 3380-3385.

[15] J. W. Polderman and J. C. Willems, Introduction to Mathematical Systems Theory: A Behavioral Approach, Springer-Verlag New York, 1998.

[16] I. W. Sandberg, On the $L_{2}$-boundedness of solutions of nonlinear functional equations, Bell Syst. Tech. J., 43 (1964), pp. 1581-1599.

[17] J. M. A. Scherpen and A. J. Van der Schaft, Normalized coprime factorizations and balancing for unstable nonlinear systems, International Journal of Control, 60 (1994), pp. 1193-1222.

[18] E. D. Sontag, Smooth stabilization implies coprime factorization, IEEE Trans. on Automatic Control, 34 (1989), pp. 435-443.

[19] A. R. Teel, Connections between razumikhin-type theorems and the ISS nonlinear small gain theorem, IEEE Trans. on Automatic Control, 43 (1998), pp. 960-964.

[20] J. C. Willems, Paradigms and puzzles in the theory of dynamical systems, IEEE Trans. on Automatic Control, 36 (1991), pp. 259-294

[21] G. Zames, Nonlinear operators for system analysis, Tech. Rept. 370, M.I.T. Research Laboratory of Electronics, Cambridge, Mass., 1960.

[22] G. Zames, Functional analysis applied to nonlinear feedback systems, IEEE Trans. on Circuit Theory, 10 (1963), pp. 392-404.

[23] G. Zames, On the input-output stability of time-varying nonlinear feedback systems-part I and II, IEEE Trans. on Automatic Control, 11 (1966), pp. 228-238 and 465-476.

[24] Q. Zheng and E. Zafiriou, A local form of small gain theorem and analysis of feedback volterra systems, IEEE Trans. on Automatic Control, 44 (1999), pp. 635-640. 